\title{
Glucoamylase (exo-1,4- $\alpha$-D-glucan glucanohydrolase, EC 3.2.1.3) is the major starch-degrading enzyme secreted by the phytopathogenic fungus Colletotrichum gloeosporioides
}

\author{
Darren R. Krause, $†$ Christopher J. Wood and Donald J. Maclean* \\ Department of Biochemistry, University of Queensland, St Lucia, Queensland 4072, Australia
}

(Received 18 April 1991; revised 24 June 1991; accepted 5 July 1991)

\begin{abstract}
Activity assays based on selective product detection were used to identify the classes of amylase present in culture filtrates of the phytopathogen Colletotrichum gloeosporioides. When transferred from a glucose medium to a medium containing starch as sole carbohydrate, $C$. gloeosporioides secreted amylolytic activity into liquid shake cultures starting 2-3 d after inoculation, and produced levels of activity up to $0.45 \mathrm{IU} \mathrm{ml}^{-1}$ (assayed by the p-hydroxybenzoic acid hydrazide - PAHBAH - reducing sugar method), about 20 times higher than the activity in comparable glucose-grown controls, suggesting that starch (or a starch degradation product) was required to induce amylase synthesis. In four separate experiments, the ratio of total reducing sugar production (assayed by the PAHBAH method) to glucose production (assayed by the glucose-specific glucose oxidase - GO-method) was about 1.0 in most samples of culture filtrate, clearly demonstrating that the major amylolytic enzyme secreted by C. gloeosporioides is a glucoamylase (exo-1,4- $\alpha$-D-glucan glucanohydrolase, EC 3.2.1.3, synonym amyloglucosidase). On one occasion only (a $7 \mathrm{~d}$ sample in one experiment) was evidence obtained for $\alpha$-amylase (endo-1,4$\alpha$-D-glucan glucanohydrolase, EC 3.2.1.1) also being present in the culture filtrate (PAHBAH/GO ratio 1.37); the presence of $\alpha$-amylase in this sample was confirmed by native polyacrylamide gel electrophoresis using a starch-iodine activity stain.
\end{abstract}

\section{Introduction}

The amylase family of enzymes has been well characterized through the study of various industrial nonphytopathogenic fungi (e.g. Ueda, 1981; Sierks et al., 1989). Two major classes of starch-degrading enzymes have been identified in the fungi: $\alpha$-amylase (endo1,4- $\alpha$-D-glucan glucanohydrolase, EC 3.2.1.1) which randomly hydrolyses the 1,4- $\alpha$-D-glucosidic links between adjacent glucose residues in linear amylose chains, and glucoamylase (synonym amyloglucosidase: exo-1,4- $\alpha$-D-glucan glucanohydrolase, EC 3.2.1.3), which cleaves single glucose units from the non-reducing ends of amylose and amylopectin in a stepwise manner. Unlike the $\alpha$-amylases, most glucoamylases are also able to hydrolyse the 1,6- $\alpha$ linkages in branch points of amylopectin, but at a slower rate than the 1,4- $\alpha$ linkages.

\footnotetext{
$\dagger$ Present address: Queensland Institute of Medical Research, Bramston Terrace, Herston, Queensland 4006, Australia.

Abbreviations: GO, glucose oxidase method for the estimation of glucose; PAHBAH, $p$-hydroxybenzoic acid hydrazide method for the estimation of reducing sugars.
}

A single fungal species may secrete both $\alpha$-amylases and glucoamylases; e.g. Aspergillus oryzae is used for the commercial production of both enzymes (cf. Montenecourt \& Everleigh, 1985).

Despite extensive characterization of the amylases secreted by industrially important fungi such as Aspergillus niger (Boel et al., 1984; Ueda, 1981), Aspergillus awamori (Nunberg et al., 1984), Rhizopus oryzae (Ashikari et al., 1986) and Saccharomyces diastaticus (Yamashita et al., 1985), we are not aware of any substantial reports on the amylases secreted by phytopathogenic fungi. For example, Charya et al. (1983) studied the in vitro degradation of starch by three phytopathogenic fungi, Rhizoctonia solani, Cladosporium cladosporioides and Colletotrichum capsici, and reported that amylolytic activity in culture filtrates (assayed by a reducing sugar method) was increased by increasing the starch concentration in the medium. Based on chromatographic analysis of residual carbohydrates in culture filtrates, it was suggested that these three phytopathogens secreted $\alpha$-amylase activity (Charya et al., 1983). However, chromatographic analysis of culture filtrates may fail to reveal the direct product(s) of starch 
hydrolysis; in particular, much of the free glucose produced by extracellular amylase(s) would be taken up and utilized as a carbon source by the fungus.

In the present investigation, we used two activity assay procedures based on the detection of both reducing sugars ( $p$-hydroxybenzoic acid hydrazide method, PAHBAH; cf. Blakeney \& Mutton, 1980) and glucose (glucose oxidase method, GO; cf. Trinder, 1969), to distinguish between $\alpha$-amylase and glucoamylase activities in culture filtrates of an isolate of the fungus Colletotrichum gloeosporioides (Penz.) Penz. \& Sacc. (type A), a phytopathogen limited to species of the tropical pasture legume Stylosanthes (Irwin \& Cameron, 1978). The PAHBAH assay method non-specifically detects all free reducing sugar groups, and hence detects the activity of both $\alpha$-amylase and glucoamylase; because oligosaccharides give a somewhat higher colour yield (e.g. maltotetraose gives $134 \%$ of the molar colour yield of glucose; cf. Blakeney \& Mutton, 1980; Lever, 1972), this method should be slightly more sensitive for detecting $\alpha$-amylase activity than glucoamylase activity. In contrast, the GO assay method is highly specific for free glucose, and hence should selectively detect glucoamylase activity and detect very little activity with $\alpha$-amylase. Also, an iodine staining procedure (which gives a characteristic blue complex with high-molecularmass amylose) was used to monitor the degradation of starch in culture filtrates and in polyacrylamide gels. Because the blue iodine colour is rapidly lost as the chain length of amylose is decreased, the iodine method preferentially indicates $\alpha$-amylase activity, and requires much higher activity of glucoamylase (as IU) for detection. The above three detection methods were used to explore the time course of secretion of amylolytic activity by $C$. gloeosporioides, and to identify the class(es) of enzymes secreted.

\section{Methods}

Chemicals and enzymes. Unless otherwise stated chemicals were of Analytical Reagent grade or equivalent. All enzymes were obtained from Sigma : $\alpha$-amylase from Aspergillus oryzae (Sigma type X-A, cat. no. A-0273, supplied as a powder $37 \mathrm{U} \mathrm{mg}^{-1}$ ); $\alpha$-amylase from porcine pancreas (Sigma type I-A, cat. no. A6255, supplied as a $2 \times$ crystallized suspension $27336 \mathrm{U} \mathrm{ml}^{-1}$ ); glucoamylase from $A$. oryzae (Sigma amyloglucosidase grade V, cat. no. A-9268, supplied as a crude solution $2566 \mathrm{U} \mathrm{ml}^{-1}$ ); glucose oxidase from Aspergillus niger (Sigma type V-S, cat. no. G-6891, supplied as a liquid $\sim 1000 \mathrm{U} \mathrm{ml}^{-1}$ 'essentially free of amylase, maltase, invertase and glycogenase'); peroxidase from horseradish (Sigma type I, cat. no. P-8125, supplied as a powder $\sim 80$ units $\mathrm{mg}^{-1}$ ).

Organism and growth. Colletotrichum gloeosporioides type A (isolate CS89) was kindly provided by Drs J. Irwin and S. Chakraborty, UQ-CSIRO Plant Pathology Unit, Botany Department, University of Queensland. Stock liquid cultures were maintained at $23^{\circ} \mathrm{C}$ on a reciprocating shaker $\left(60\right.$ cycles $\left.\mathrm{min}^{-1}\right)$ in the dark in $250 \mathrm{ml}$
Erlenmeyer flasks containing $100 \mathrm{ml}$ minimal medium $(2 \%, w / v$, glucose, $20 \mathrm{~mm}$-ammonium chloride, $10 \mathrm{~mm}$-sodium citrate, Czapek's mineral solution to $100 \%$, final $\mathrm{pH} 6.0$, sterilized at $121^{\circ} \mathrm{C}$ for $10 \mathrm{~min}$ ), essentially as described by Williams et al. (1984) for Penicillium expansum. For induction experiments, thick stock cultures grown for the number of days specified were filtered on one layer of Miracloth (Calbiochem) under suction, and the mycelium washed with glucosefree minimal medium. The mycelial pads were resuspended in $100 \mathrm{ml}$ glucose-free minimal medium, fragmented for $10 \mathrm{~s}$ in an Osteriser blender ('chop' setting), and $20 \mathrm{ml}$ of the suspension added to $100 \mathrm{ml}$ minimal medium containing appropriate carbon source (to a final concentration of $1 \%$, w/v, in $120 \mathrm{ml}$ ). Starch media contained Analar soluble starch (BDH), dissolved and simultaneously sterilized by autoclaving at $121{ }^{\circ} \mathrm{C}$ for $10 \mathrm{~min}$, followed by rapid cooling to prevent the slow subsequent precipitation of amylose. In all experiments, dry weights were determined (Williams et al., 1984) immediately after the fragmentation step, and the concentration of the mycelial suspension adjusted such that culture flasks finally contained $1 \mathrm{mg}$ dry weight $\mathrm{ml}^{-1}$ at the time of inoculation (this heavy inoculum was used to obtain prolific growth at early times after inoculation); however, dry weight measurements were not made subsequently, because the heavy initial inoculum generally resulted in thick mycelial growth which could not be sampled reproducibly for mycelial matter 2-3 days after inoculation. During induction experiments, culture flasks were incubated at $27{ }^{\circ} \mathrm{C}$ in a gyrotary culture shaker at 120 cycles $\min ^{-1}$ in the dark. Culture filtrates were obtained from $3 \mathrm{ml}$ aliquots of fungal suspension taken daily or as specified; duplicate aliquots of all samples were assayed for residual starch or amylase activity, and mean values are presented.

lodine assay of residual starch in culture media. Residual starch was assayed directly in aliquots of the crude culture filtrate, using an iodine method adapted from Smith \& Roe (1949) and Gibson \& Paleg (1975). A stock $\mathrm{I} / \mathrm{KI}$ reagent $(0.6 \mathrm{~g}$ iodine crystals, $6 \mathrm{~g}$ potassium iodide, to $100 \mathrm{ml}$ with water) was diluted immediately before use $(2.5 \mathrm{ml}$ stock to $100 \mathrm{ml}$ with water) to give a working reagent. Aliquots of culture filtrate (usually $0.1 \mathrm{ml}$ ) were made up to $1 \mathrm{ml}$ with water, to which were added $0.75 \mathrm{ml} \mathrm{I} / \mathrm{KI}$ working reagent, $0.2 \mathrm{ml} 2 \mathrm{M}-\mathrm{HCl}$ and $5 \mathrm{ml}$ water, and the absorbance read at $620 \mathrm{~nm}$ within $10 \mathrm{~min}$.

Assay of amylolytic activity. Prior to assay of amylolytic activity, crude culture filtrates were passed through a Sephadex G25M column (PD10, Pharmacia, $9 \mathrm{ml}$ bed volume) to remove sugars and other small molecules. Both the PAHBAH and GO methods used the following incubation conditions. To $0.2 \mathrm{ml}$ of $0.5 \%$ soluble starch (Analar, BDH, dissolved by autoclaving in $50 \mathrm{~mm}$-sodium maleate buffer $\mathrm{pH} 6.5$ at $121^{\circ} \mathrm{C}$ for $1-2 \mathrm{~min}$ followed by rapid cooling on ice) was added $0.6 \mathrm{ml}$ of water, and the reaction started by addition of $0.2 \mathrm{ml}$ enzyme sample. After incubation at $37^{\circ} \mathrm{C}$ for $10-60 \mathrm{~min}$ (to fit within the linear range of the assay according to the activity of the sample), the reaction was stopped by either (i) immersing the assay tube in boiling water for $5 \mathrm{~min}$ (GO assay), or (ii) adding $2.5 \mathrm{ml}$ colour reagent (PAHBAH assay). Zero time controls were set up by stopping the reaction immediately after adding the enzyme sample; both the PAHBAH and GO methods used glucose $(0-0.5 \mu \mathrm{mol})$ as standard. The PAHBAH colour reagent was prepared and the colour developed and the absorbance read at $415 \mathrm{~nm}$ as described by Blakeney \& Mutton (1980); note that phosphate buffer is incompatible with this reagent. For the GO assay, $2 \mathrm{ml} \mathrm{GO}$ colour reagent (modified from Trinder, 1969) was added, and incubated at $37^{\circ} \mathrm{C}$ for $30 \mathrm{~min}$, and the absorbance read at $510 \mathrm{~nm}$ [colour reagent : $0.75 \mathrm{ml}$ glucose oxidase (Sigma G-6891), $3 \mathrm{mg}$ peroxidase (Sigma P-8125), $5 \mathrm{mg}$ 4-aminoantipyrine, $90 \mathrm{mg} p$-hydroxybenzoic acid, $100 \mathrm{mg}$ sodium azide, in $50 \mathrm{~mm}$-sodium phosphate pH 7.0, final volume $100 \mathrm{ml}$, prepared freshly]. Enzyme samples gave near-linear rates up to absorbance values of $\sim 2$, for both the PAHBAH and GO methods. Activities are expressed as International Units (IU), calculated as $\mu \mathrm{mol}$ glucose or its equivalent produced $\mathrm{min}^{-1}$. 
Polyacrylamide gel electrophoresis (PAGE). PAGE of amylase preparations was carried out under non-denaturing (native) conditions using $7 \%$ polyacrylamide in $1 \mathrm{~mm}$ vertical slabs with an LKB 2050 Midget electrophoresis unit; running buffer $0.01 \mathrm{M}$-Tris, $0.02 \mathrm{M}$ glycine, $\mathrm{pH} \mathrm{8.7}$. Electrophoresis was carried out at $200 \mathrm{~V}$ for about $2 \mathrm{~h}$. Amylase activity was visualized (stain modified from Kakefuda \& Duke, 1984) by first equilibrating gels in $50 \mathrm{~mm}$-maleate buffer $\mathrm{pH} 6.5$ for $5 \mathrm{~min}$, followed by immersion in $2 \%(\mathrm{w} / \mathrm{v})$ starch (dissolved in the maleate buffer) for $15 \mathrm{~min}$ at room temperature. The gels were incubated for $10 \mathrm{~min}$ at $45^{\circ} \mathrm{C}$ in the same buffer without starch, then placed on a piece of Miracloth soaked in working $\mathrm{I} / \mathrm{KI}$ reagent (prepared as described above for assay of residual starch in culture media). The gels were photographed after incubation at room temperature for 5-10 min to allow the blue background colour to develop.

\section{Results}

Time course of induction of amylolytic activity in liquid shake cultures

Four separate experiments were set up to explore the time-course of induction of secreted amylolytic activity during growth of $C$. gloeosporioides on a $1 \%(\mathrm{w} / \mathrm{v})$ starch medium: experiments 1, 3 and 4 used mycelial inoculum grown on a glucose medium, whereas experiment 2 used inoculum grown on a starch medium. Figs 1 and 2 present data from experiments 1 and 2, using the iodine assay to estimate the rate of degradation of highmolecular-mass starch in culture filtrates (Fig. 1), and the PAHBAH reducing sugar method to measure enzymic activity (Fig. 2). Comparison of Figs 1 and 2 shows that starch was degraded concomitantly with the accumulation of amylase activity in the medium. Indeed, amylase activity continued to accumulate in the medium (experiment 1) after total loss of the iodine-blue colour, suggesting either that lower-molecular-mass amylose oligomers (which have lost iodine-staining capacity) still present in the medium possessed inducing activity, or that the fungus remained in the induced state after degradation of the initial inducing substrate. In all four experiments, very little amylolytic activity was detected in culture filtrates of control flasks containing glucose as sole carbohydrate source (e.g. Fig. 2 for experiment 1 ).

The earlier onset of starch degradation and amylase secretion observed in experiment 2 compared to experiment 1 (Figs 1 and 2), is attributed to the inoculum having been grown previously on starch medium, hence requiring less time for full induction of amylase secretion to occur after transfer to fresh starch medium. In further experiments (data not presented) to explore conditions for the induction of amylase synthesis, $1 \%(\mathrm{w} / \mathrm{v})$ $\beta$-cyclodextrin was substituted for starch as carbohydrate source; however, no appreciable growth or secreted amylolytic activity was observed, in contrast to Aspergillus oryzae (Jodal et al., 1984) and various yeasts (De Mot

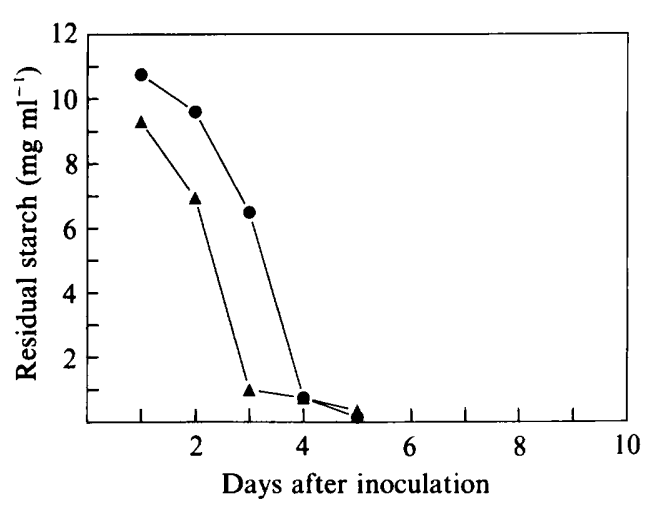

Fig. 1. Assays of residual starch (estimated by the iodine method) in culture filtrates taken after growth of $C$. gloeosporioides in liquid medium containing $1 \%(w / v)$ soluble starch. Experiment $1(\bullet)$ used inoculum previously grown on glucose as sole carbohydrate for $\sim 7 \mathrm{~d}$. Experiment $2(\boldsymbol{A})$ used inoculum previously grown on starch as sole carbohydrate for $\sim 7 \mathrm{~d}$.

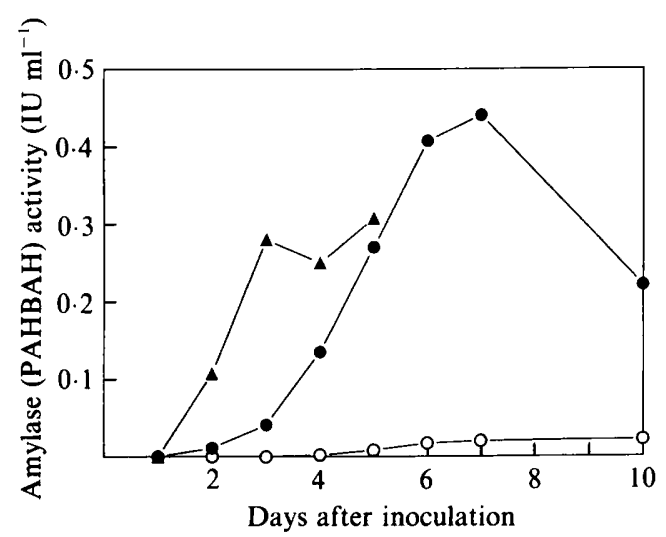

Fig. 2. Amylolytic activity in culture filtrates taken from C. gloeosporioides after growth in liquid medium containing $1 \%(\mathrm{w} / \mathrm{v})$ soluble starch, estimated by the PAHBAH reducing sugar method. Symbols and growth conditions are described in the legend to Fig. 1; open circles represent samples from a control (experiment 1) grown on starch-free medium containing $1 \%(\mathrm{w} / \mathrm{v})$ glucose as sole carbohydrate source.

\& Verachtert, 1986) which secrete $\alpha$-amylase or glucoamylase or both when grown with cyclodextrin as sole carbohydrate. Maltose ( $1 \%$, w/v) supported growth, but did not induce the secretion of amylolytic activity as reported for other fungi (e.g. De Mot \& Verachtert, 1986). HPLC analysis of culture filtrates using the carbohydrate analysis system described by McKay et al. (1987) demonstrated that after growth on starch media, culture filtrates contained neutral compounds with the retention times of glucose and starch fragments. HPLC analysis of the reaction products from activity assays showed that the major product of starch hydrolysis was a neutral compound with the retention time of glucose, 
indicating that considerable exoglucanase activity appeared to be present.

In the three experiments (1, 3 and 4$)$ which used inoculum grown on glucose, the age and physiological state of the inoculum affected the amount of amylase secreted. Thus in experiment 1 using relatively young inoculum (subcultured $7 \mathrm{~d}$ previously), the amount of amylase secreted was far greater than in experiments 3 and 4 using older inocula (respectively subcultured 15 and $23 \mathrm{~d}$ previously, cf. Table 2). Although this can primarily be attributed to more rapid initial mycelial growth from a younger inoculum, it is worth noting that C. gloeosporioides is dimorphic when grown in axenic culture (Slade et al., 1987), and older liquid cultures frequently revert to a predominantly unicellular conidial form (observed in experiments 3 and 4), compared to the predominantly filamentous mycelial form of fresh cultures (observed in experiments 1 and 2). These observations suggest that secretion of amylolytic activity may be controlled by the physiological state of the inoculum, and that the filamentous form of the fungus is required for maximum amylase secretion. Although lower amylolytic activities were measured in experiments 3 and 4 , critical experiments to determine whether the conidial form of the fungus is capable of amylase secretion were not pursued, because the main purpose of the above experiments was to determine which classes of amylase were secreted, and whether this might be affected by the pretreatment of inoculum or morphological form of the fungus.

\section{Comparison of PAHBAH and GO methods for assay of amylolytic activity}

To verify the approach of using both the PAHBAH and GO assay methods to define the substrate specificity (i.e. cleavage sites) of the amylolytic enzyme(s) secreted by C. gloeosporioides, commercially available preparations of specific fungal amylases were assayed by both methods (Table 1). Because both assay protocols were identical (except for colorimetric detection of product) and used glucose as standard, glucoamylase should give a theoretical PAHBAH/GO ratio of $1 \cdot 0$, a value to which the commercial glucoamylase from Aspergillus oryzae approximated $(\sim 1 \cdot 2)$; the slightly higher value could be caused by contaminating $\alpha$-amylases, as $A$. oryzae is known to produce both enzymes. In contrast, the commercial preparations of $\alpha$-amylase gave a $\mathrm{PAHBAH} / \mathrm{GO}$ ratio of 28 , approaching the theoretical value of infinity (higher values were obtained in other experiments not presented here, ranging from 73 for porcine pancreatic $\alpha$-amylase, to 625 for another batch of Aspergillus oryzae $\alpha$-amylase). The large difference in
Table 1. Comparative activity of commercially available fungal amylases, determined by the rate of formation of either reducing sugar (PAHBAH assay method) or glucose ( $G O$ assay method)

Each amylase sample was diluted to a concentration within the linear activity range for both assay methods.

\begin{tabular}{lcc}
\hline \hline \multicolumn{1}{c}{ Assay method } & $\begin{array}{c}\alpha \text {-Amylase* } \\
\left(\mathrm{IU} \mathrm{ml}^{-1}\right)\end{array}$ & $\begin{array}{c}\text { Glucoamylase } \\
\left(\mathrm{IU} \mathrm{ml} \mathrm{m}^{-1}\right)\end{array}$ \\
\hline Reducing sugar formation (PAHBAH) & 0.042 & 0.034 \\
Glucose formation (GO) & 0.0015 & 0.03 \\
PAHBAH/GO ratio & 28 & $1 \cdot 2$ \\
\hline \hline
\end{tabular}

* $\alpha$-Amylase from Aspergillus oryzae (Sigma, type X-A).

$†$ Glucoamylase from Aspergillus oryzae (Sigma, type A-9268).

Table 2. Ratio of amylolytic activities (PAHBAH/GO ratio), as assayed by the PAHBAH (reducing sugar) and GO (glucose-specific) methods, in samples of culture filtrate taken during growth of $C$. gloeosporioides in liquid shake culture

Results are shown from four independent experiments, with cultures inoculated, incubated and sampled as described in Figs 1 and 2. Experiments 1 and 2 are the same as those described in Fig. 1 (using 7-d-old mycelium as inoculum); experiments 3 and 4 were set up similarly but used 15-23-d-old mycelium as inoculum. A blank space indicates not determined.

\begin{tabular}{ccccc}
\hline \hline \multirow{2}{*}{$\begin{array}{c}\text { Days after } \\
\text { inoculation }\end{array}$} & \multicolumn{4}{c}{ PAHBAH/GO ratio (PAHBAH activity)* } \\
\cline { 2 - 5 } & Expt 1 & Expt 2 & Expt 3 & Expt 4 \\
\hline 2 & $0.83(0.01)$ & $0.86(0.11)$ & & \\
3 & $0.85(0.04)$ & $1.00(0.28)$ & & \\
4 & $0.82(0.14)$ & $0.89(0.25)$ & $0.79(0.09)$ & $0.92(0.03)$ \\
5 & $0.95(0.27)$ & $0.74(0.31)$ & $0.90(0.12)$ & $0.77(0.03)$ \\
6 & $1.01(0.41)$ & & $0.83(0.15)$ & $0.84(0.04)$ \\
7 & $\mathbf{1 . 3 7 ( 0 . 4 4 ) \dagger}$ & & $1.00(0.22)$ & $0.96(0.05)$ \\
10 & $0.92(0.22)$ & & & \\
\hline
\end{tabular}

* Culture filtrates were assayed by both the PAHBAH and GO assay methods as explained in Table 1 , and activities expressed as IU ml-1 prior to calculating PAHBAH/GO activity ratios. Values in parentheses are the results of the PAHBAH assays in IU ml-1 culture filtrate.

† This result is emboldened to indicate it as the only sample with a ratio markedly above $1 \cdot 0$.

PAHBAH/GO ratio between glucoamylase and $\alpha$-amylase clearly demonstrates that this ratio can be used to distinguish exoglucanases from endoglucanases.

The PAHBAH/GO ratio was determined in samples from all four time-course experiments described in the previous section (cf. Figs 1 and 2 for experiments 1 and 2 ), and the full results are presented in Table 2. Except for the $7 \mathrm{~d}$ sample in experiment 1 , this ratio was $\sim 1.0$ or less for all samples in all experiments (ratio ranging from 0.74 to 1.01 ), including experiments 3 and 4 in which 


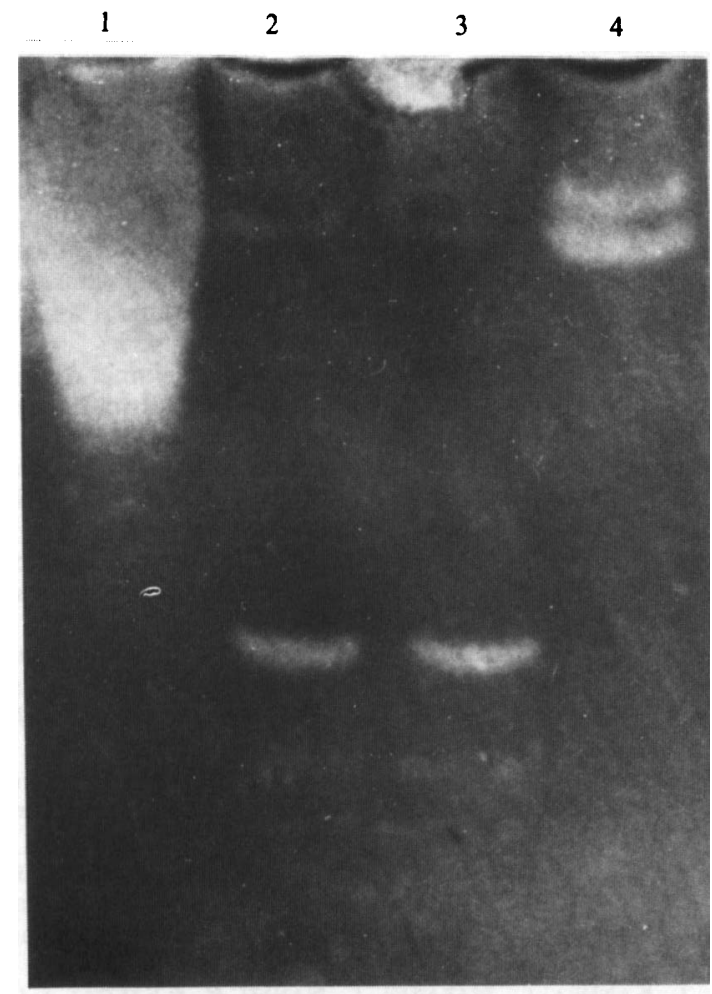

Fig. 3. Photograph of a polyacrylamide gel showing banding patterns for amylase activity using the iodine stain. The activity of samples loaded on the gel was determined by the PAHBAH assay method. Lane 1, 0.16 IU $\alpha$-amylase from porcine pancreas (Sigma type I-A); lanes 2 and 3, glucoamylase from Aspergillus oryzae (Sigma type A-9268), lane 2, $0.065 \mathrm{IU}$, lane $3,0 \cdot 13 \mathrm{IU}$; lane $4,2 \mu \mathrm{l}$ of sixfold concentrate (Amicon pressure cell) of culture filtrate from C. gloeosporioides grown on starch medium for $7 \mathrm{~d}(0.001$ IU activity loaded, PAHBAH/GO activity ratio of $1 \cdot 37$ ).

much of the fungus was in the conidial rather than the mycelial form; these values approximate to the ratio of 1.0 predicted for glucoamylase activity (ratios slightly less than 1.0 are attributed to the small deviations from linearity of the activity assays noted in Methods). A ratio of $\sim 1.0$ was also observed for the low levels of secreted activity after growth of $C$. gloeosporioides on starch-free $(1 \%, w / v$, glucose) medium (data not presented). The PAHBAH/GO ratio of 1.37 obtained for the $7 \mathrm{~d}$ sample in experiment 1 suggested that most of the amylase activity in this sample was a glucoamylase, with a small proportion of $\alpha$-amylase.

\section{Electrophoretic analysis of $\alpha$-amylase in culture filtrate}

PAGE was used to confirm that the PAHBAH/GO ratio of 1.37 given by the $7 \mathrm{~d}$ sample of experiment 1 was caused by the presence of $\alpha$-amylase together with the predominant glucoamylase. The $7 \mathrm{~d}$ sample of culture filtrate lost $\sim 75 \%$ of its initial PAHBAH activity during storage on ice for $44 \mathrm{~h}$ prior to electrophoresis, but lost even more GO activity during this time, resulting in a final PAHBAH/GO ratio of $1 \cdot 82$. Native PAGE of a sixfold concentrate (Amicon pressure cell) of the stored sample, followed by starch-iodine staining, revealed two bands of low mobility (Fig. 3, lane 4). On the same gel, porcine pancreatic $\alpha$-amylase also showed low mobility (lane 1) compared to the highly mobile $A$. oryzae glucoamylase (lanes 2 and 3 ). The selectivity of the starch-iodine stain for $\alpha$-amylase is demonstrated by the very intense (and smeared) band given by the purified porcine $\alpha$-amylase ( $0.16 \mathrm{IU}$, PAHBAH assay), compared to the much fainter band given by a similar number of PAHBAH units of activity of the $A$. oryzae glucoamylase (0.13 IU). However, only 0.001 IU (PAHBAH assay) of activity from the $C$. gloeosporioides culture filtrate (Fig. 3, lane 4), was sufficient to give faint bands, comparable in intensity to the 0.13 IU of authentic glucoamylase (lane $3)$. Because the glucoamylase activity in the $7 \mathrm{~d} C$. gloeosporioides culture filtrate would have been too low to give a detectable band in the gel, it follows that the activity bands seen in this sample (lane 4) represent authentic $\alpha$-amylase activity.

\section{Discussion}

The phytopathogen Colletotrichum gloeosporioides type A secreted extracellular amylolytic activity into culture filtrates when grown on starch medium in axenic culture; because very little activity was secreted in glucose medium, it appears that starch (or a starch degradation product, perhaps present in the Analar soluble starch used in growth media) is required to induce the synthesis of starch-degrading enzymes by this fungus. Neither $\beta$-cyclodextrin nor maltose could substitute for soluble starch as an inducer of amylase synthesis.

This investigation demonstrated that the major amylolytic enzyme secreted by $C$. gloeosporioides was a glucoamylase (exo-1,4- $\alpha$-D-glucan glucanohydrolase, EC 3.2.1.3). This was achieved using two colorimetric methods to distinguish between the reaction products of glucoamylase (glucose, detected by both the GO and PAHBAH methods) and $\alpha$-amylase (liberation of reducing sugar groups on oligosaccharides, detected by the PAHBAH method but not the GO method). Commercial preparations of amylases gave PAHBAH/GO ratios approaching the theoretical values of 1.0 for glucoamylases, and infinity for $\alpha$-amylase. Thus, the ratio of 0.8-1.0 for most culture filtrates of $C$. gloeosporioides clearly indicated the presence of glucoamylase and the virtual absence of $\alpha$-amylase.

In only one sample taken during the four time-course experiments did the culture filtrate of starch-induced 
C. gloeosporioides exhibit a PAHBAH/GO ratio appreciably above 1.0 (ratio of 1.37 ), indicating that $\alpha$-amylase was present together with glucoamylase. The presence of $\alpha$-amylase in this sample was confirmed by the starchiodine stain after native PAGE. The absence of appreciable $\alpha$-amylase activity in most samples during growth of the fungus in starch medium, and its detection only in one experiment and then only $7 \mathrm{~d}$ after inoculation, was the converse of predictions prior to starting this investigation. Thus, Charya et al. (1983), on the basis of chromatographic analysis of the carbohydrates present in culture filtrates, previously reported that a number of phytopathogens including Colletotrichum capsici secreted $\alpha$-amylase when grown on starch medium. Further research is required to determine whether secretion of glucoamylase as the major amylolytic enzyme is a general characteristic of phytopathogens, or is restricted to a particular class of phytopathogens which include $C$. gloeosporioides. Such research should use techniques which unequivocally distinguish glucoamylase from $\alpha$-amylase, e.g. by determining the PAHBAH/GO ratio. The reason(s) for the low and inconsistent secretion of $\alpha$-amylase by $C$. gloeosporioides are unknown, and were not addressed in the present investigation.

$\alpha$-Amylase is known to act synergistically with glucoamylase (e.g. for Aspergillus sp. K-277; Abe et al., 1988) by first degrading complex starch substrates to smaller glucan oligomers, which then present many nonreducing ends for glucoamylase to work on; it was therefore predicted that appreciable $\alpha$-amylase activity would be secreted prior to glucoamylase. It thus appears that the glucoamylase of $C$. gloeosporioides is responsible for most of the degradation of complex starch substrates, perhaps with the assistance of trace $\alpha$-amylase activity. It remains for future research to determine whether the glucoamylase of $C$. gloeosporioides resembles the glucoamylase G1 of Aspergillus awamori in having high 1,6- $\alpha$ debranching activity which enables it to degrade raw starch (Ueda, 1981), or whether low activities of secreted $\alpha$-amylase (undetected by the PAHBAH/GO ratio) are sufficient to break up raw starch enough to permit its complete degradation by glucoamylase. It also remains for future research to investigate the biological role of glucoamylase during pathogenesis by $C$. gloeosporioides (e.g. to obtain soluble carbohydrate from starch grains in host tissues).

Financial support given by the University of Queensland Special Projects Grants Scheme, and technical assistance from M. Sweet and S. Nolan, is gratefully acknowledged. We thank Drs J. M. Manners, S. Chakraborty and J. A. G. Irwin, CSIRO/UQ Plant Pathology Unit, and Dr R. Henry, Queensland Wheat Research Institute, for advice and helpful discussions.

\section{References}

Abe, J.-I., Nakajima, K., Nagano, H., Hizukuri, S., \& Obata, K. (1988). Properties of the raw-starch digesting amylase of Aspergillus sp. K-27: a synergistic action of glucoamylase and alpha-amylase. Carbohydrate Research 174, 85-92.

Ashikari T., Nakamura, N., Tanaka, Y., Kiuchi, N., Shibano, Y., TanaKa, T., Amachi, T. \& Yoshizumi, H. (1986). Rhizopus rawstarch-degrading glucoamylase: its cloning and expression in yeast. Agricultural and Biological Chemistry 50, 957-964.

Blakeney, A. B. \& Mutton, L. L. (1980). A simple colorimetric method for the determination of sugars in fruit and vegetables. Journal of the Science of Food and Agriculture 31, 889-897.

Boel, E., SVensson, H., NorRIs, K. \& FIIL, N. (1984). Glucoamylases G1 and G2 from Aspergillus niger are synthesised from two different but closely related mRNAs. EMBO Journal 3, 1097-1102.

Charya, M., Reddy, S. \& Kumar, B. (1983). $\alpha$-Amylase production in relation to assimilation of starch by three fungi. Proceedings of the National Academy of Sciences of India 53, 19-25.

DE MOT, R. \& VERACHTERT, H. (1986). Enhanced production of extracellular $\alpha$-amylase and glucoamylase by amylolytic yeasts using $\beta$-cyclodextrin as carbon source. Applied Microbiology and Biotechnology 24, 459-462.

Gibson, R. A. \& Paleg, L. G. (1975). Further experiments on the $\alpha$-amylase-containing lysosomes of wheal aleurone cells. Australian Journal of Plant Physiology 2, 41-49.

IRWIN, J. A. G. \& CAMERON, D. F. (1978). Two diseases of Stylosanthes spp. caused by Colletotrichum gloeosporioides in Australia, and pathogenic specialization within one of the causal organisms. Australian Journal of Agricultural Research 29, 305-317.

Jodal, I., Kandra, L., Harangi, J., Nanasi, P. \& SzeJTLI, J. (1984). Hydrolysis of cyclodextrin by Aspergillus oryzae $\alpha$-amylase. Starch 36, 140-143.

KAKEFUDA, G. \& DUKe, S. (1984). Electrophoretic transfer as a technique for the detection and identification of plant amylolytic enzymes in polyacryamide gels. Plant Physiology 75, 278-280.

LEVER, M. (1972). A new reaction for colorimetric determination of carbohydrates. Analytical Biochemistry 47, 273-279.

MCKaY, D. B., Tanner, G. P., Maclean, D. J. \& ScotT, K. J. (1987). Detection of polyols and sugars by cuprammonium ion in the presence of strong base. Analytical Biochemistry 165, 392-398.

MONTENeCOURT, B. \& Eveleigh, D. (1985). Fungal carbohydrases: amylases and cellulases. In Gene Manipulations in Fungi, pp. 491-512. Edited by J. W. Bennet \& L. L. Lasure. New York \& London: Academic Press.

Nunberg, J., Meade, J., Cole, G., Lawyer, F., McCabe, P., Schweikart, V., Tal, R., Wittman, V., Flatgaard, J. \& Innis, M. (1984). Molecular cloning and characterization of the glucoamylase gene of Aspergillus awamori. Molecular and Cellular Biology 4, 2306-2315.

Sierks, M. R., Ford, C., Reilly, P. J. \& Svensson, B. (1989). Sitedirected mutagenesis at the active site Trp120 of Aspergillus awamori glucoamylase. Protein Engineering 2, 621-625.

Slade, S. J., Harris, R. F., Smith, C. S. \& Andrews, J. H. (1987). Microcycle conidiation and spore-carrying capacity of Colletotrichum gloeosporioides on solid media. Applied and Environmental Microbiology 53, 2106-2110.

SMITH, B. W. \& RoE, J. H. (1949). A photometric method for the determination of $\alpha$-amylase in blood and urine, with use of the starch-iodine colour. Journal of Biological Chemistry 179, 53-59.

TRINDER P. (1969). Determination of blood glucose using glucose oxidase with an alternative oxygen acceptor. Annals of Clinical Biochemistry 6, 24-27.

UEDA, S. (1981). Fungal glucoamylases and raw starch digestion. Trends in Biochemical Sciences 6, 89-90.

Williams, A. M., Maclean, D. J. \& ScotT, K. J. (1984). Cellular location and properties of invertase in mycelium of Puccinia graminis. New Phytologist 98, 451-463.

Yamashita, I., SUZUKI, K. \& FUKUI, S. (1985). Nucleotide sequence of the extracellular glucoamylase gene $S T A I$ in the yeast Saccharomyces diastaticus. Journal of Bacteriology 161, 567-573. 